\title{
The proliferation of nano journals
}

To the Editor - Twenty-five years after the letter announcing the discovery of fullerenes ${ }^{1}$ was published in Nature, I am struck by the sheer number of journals that have been launched for the primary purpose of publishing research papers in various areas of nanoscience and nanotechnology. Many scientometric studies have focused on citation patterns or the geographical distribution of authorship ${ }^{2-4}$. This article will quantify the growth over time in the number of nanotechnology journals using three databases published by Thomson Reuters: the Science Citation Index, the Journal Citation Reports (JCR) and the Web of Knowledge.

The first journal devoted exclusively to nanoscale science and technology, Nanotechnology, was launched by Institute of Physics Publishing in July 1990. Its first volume contained just 21 research papers, compared with about 1,000 papers per year at present. There has been a similarly dramatic increase in the number of nanotechnology journals. A survey of periodicals - including journals, book series (excluding those derived from conferences), magazines and newsletters that contain substantial articles - has revealed that 270 different nanotechnology titles have been published (see Supplementary Information). Twentytwo of these titles are open access journals.

Note that these figures do not include general science journals (such as Nature and Science) or discipline-specific journals (such as Angewandte Chemie, Applied Physics Letters and many others) that also publish papers on nanoscience and nanotechnology. In fact, a search of the Science Citation Index using the 'Georgia Tech phase I' search strategy ${ }^{5}$ yields at least one hit for almost half of the journals covered by the index in 2009 (3,658 of 7,387), although a few per cent were undoubtedly 'false positives' (data not shown).

Yearly data for the launch of new journals with a known publication history, and excluding mere title changes, yields exponential growth beginning in around 1997 with respect to the cumulative total of titles that are still 'currently published' (and also with respect to the cumulative number of launches; Fig. 1). By 1998, only 15 nanotechnology journals had been launched, whereas a total of 165 had been launched by 30 October 2010 . Of these, 142 are still producing issues. The proportional increase in the number of nanotechnology journals over time is similar to the increase in the number of research papers on

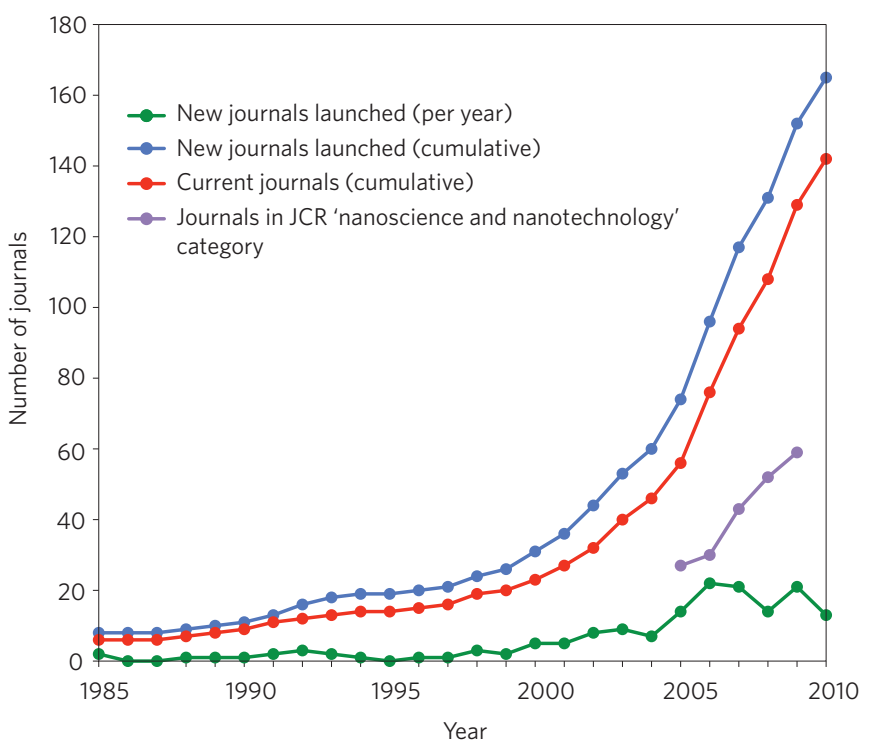

Figure 1 | Growth of the nano literature. The green line shows the number of new nanotechnology journals launched each year from 1985 to the present; the blue line is a cumulative tally of all nanotechnology journals (including those that have ceased publication); the red line includes only current journals (defined as journals that have published at least one issue dated '2009' or '2010' as of 30 October 2010). Only journals that publish original research papers are included; newsletters, book series, magazines and conference series publications have been excluded. The purple line shows the number of journals in the 'nanoscience and nanotechnology' category of the JCR.

nanotechnology (which also includes research papers in other journals ${ }^{6}$ ).

The 'nanoscience and nanotechnology' journal category first appeared in the JCR in 2005, and the number of journals in this category has more than doubled, from 27 in 2005, to 59 in 2009 (Fig. 1). Of the dozens of journal categories listed in the JCR, only two showed such a large proportional increase from 2005 to 2009: nursing (32 to 72 titles) and remote sensing (10 to 21 titles).

Of the 175 current and defunct journal titles (Tables S1a and S1d in Supplementary Information), only $71(\sim 41 \%)$ have ever been covered by the Web of Knowledge database. Whereas coverage of most of them commenced with the first issue, coverage of the first nano journal, Nanotechnology, did not start until the first issue of volume 5 , which suggests that papers in the earlier volumes did not have enough citations to be included. As Feynman's classic 1959 lecture proved ${ }^{7}$, the price you pay for being 'ahead of your time' is that it may be a while before others cite your work, and it is likely that many of the recently launched nanotechnology journals will be added to the Web of Knowledge as their citation counts grow.

As research fields mature, more qualifying adjectives and suffixes tend to appear in the titles of journals as broad-based publications are joined by titles of increasing specialization. Witness, for example, the recent launches including the Journal of Nanoneuroscience, Nano Communication Networks and Cancer Nanotechnology. And with more than a dozen other new titles in the pipelines, the number of nanotechnology journals looks set to continue growing.

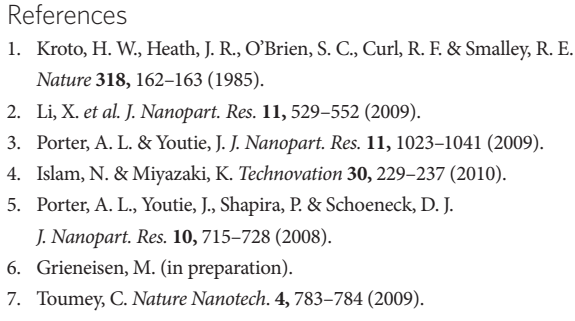
www.nature.com/naturenanotechnology.

\section{Michael L. Grieneisen}

Department of Land, Air and Water Resources, AGIS Lab of Minghua Zhang, University of California, Davis, California 95616, USA. e-mail: biblioforhire@yahoo.com

Published online: 21 November 2010 